\title{
Monitoring a lab-scale wurster type fluidized bed process by electrical capacitance tomography
}

DOI:

10.1016/j.flowmeasinst.2017.09.005

\section{Document Version}

Accepted author manuscript

Link to publication record in Manchester Research Explorer

\section{Citation for published version (APA):}

Che, HQ., Wu, M., Ye, JM., Yang, W., \& Wang, HG. (2018). Monitoring a lab-scale wurster type fluidized bed process by electrical capacitance tomography. Flow Measurement and Instrumentation, 62, 223-234.

https://doi.org/10.1016/j.flowmeasinst.2017.09.005

\section{Published in:}

Flow Measurement and Instrumentation

\section{Citing this paper}

Please note that where the full-text provided on Manchester Research Explorer is the Author Accepted Manuscript or Proof version this may differ from the final Published version. If citing, it is advised that you check and use the publisher's definitive version.

\section{General rights}

Copyright and moral rights for the publications made accessible in the Research Explorer are retained by the authors and/or other copyright owners and it is a condition of accessing publications that users recognise and abide by the legal requirements associated with these rights.

\section{Takedown policy}

If you believe that this document breaches copyright please refer to the University of Manchester's Takedown Procedures [http://man.ac.uk/04Y6Bo] or contact uml.scholarlycommunications@manchester.ac.uk providing relevant details, so we can investigate your claim.

\section{OPEN ACCESS}




\title{
Monitoring a Lab-scale Wurster Type Fluidized Bed Process by Electrical Capacitance Tomograph*
}

\author{
H.Q. Che ${ }^{1,2}$, M. Wu ${ }^{1,2}$, J.M. Ye ${ }^{1}$, W.Q. Yang ${ }^{3}$ and H.G. Wang ${ }^{1,2}$ \\ ${ }^{1 .}$ Institute of Engineering Thermophysics, Chinese Academy of Sciences, Beijing 100190, China \\ 2. University of Chinese Academy of Sciences, Beijing 100049, China \\ 3. School of Electrical and Electronic Engineering, University of Manchester, Manchester, M13 9PL, UK
}

\begin{abstract}
In this research, two types of electrical capacitance tomography (ECT) sensors, i.e. 12-4-8 combined electrodes and 8-8 dual planes sensors, were designed and used to monitor the gas-solids flow inside a Wurster type fluidized bed. For the 12-4-8 combined electrodes sensor, the measurement was conducted synchronously both inside and outside of the tube, i.e. coating zone and annulus zone, to achieve a fully understand the gas-solids flow characteristics in the bed. For the dual planes ECT sensor, the flow through the two cross-section areas inside the Wurster tube was measured and solids velocity was calculated based on cross-correlation method. A series of test were carried out by varying the operational parameters including the gap between the Wurster tube and air distributor, fluidization air velocity and materials loading. Different flow regimes as well as the flow stability were evaluated based on the ECT measurement results. To evaluate the flow characteristics inside the bed, power spectra density (PSD) and standard deviation (SD) were applied to investigate the fluctuation characteristics in the coating and annulus zones. Experiment results indicate that ECT technology is a powerful tool to monitor the Wurster type fluidized bed process and analysis the gas-solids flow characteristics inside the bed.
\end{abstract}

Keywords: Fluidized bed, Wurster tube, Electrical capacitance tomography, Solids concentration

\section{Introduction}

Fluidized beds are widely used in pharmaceutical, chemical and food industries for particles coating, granulation and drying. In the coating process, particles are fluidized by hot air, while solution or solvent is sprayed into the same zone and contacted with the particles by dual-fluid nozzle. The particles are wetted by the solution or solvent droplets and dried by hot air for a certain time up to form a coating film on the particle's surface. The coating film helps to mask the taste of unpalatable substances and provide sustained or extended release. As a one-step process and enclosed operation, the process can be scale-up to industry scale with low cost. Depending on the desired products and the position of the spray nozzles, several types of technologies are used, including top-spray, bottom-spray and tangential spray [1]. Among them, the bottom-spray was firstly introduced by Wurster [2] and it is one type of fluidized bed with an internal vertical tube named

* Correspondence author, Tel.: 0086-10-82543140, E-mail: wanghaigang@iet.cn (H.G. Wang) 
Wurster tube above the air distributor. The air distributor blow Wurster tube was designed to control the airflow with a higher speed in the coating zone and a lower in the annulus zone, resulting in a pressure difference between the two regions. The pressure difference creates a pneumatic mass transport from the annulus zone to the coating zone through the gap between the Wurster tube and air distributor. According to the particles movement, the flow field inside the bed was divided into four different zones, namely coating, drying, annulus and transporting zone. In general, the bottom-spray is the best choice for particle coating as it can produce a superior film compared with other coating strategies $[1,3]$.

In the fluidized bed coating process with Wurster tube, the quality of product is strongly depended on the operation parameters, i.e. fluidization air velocity, inlet air temperature, the gap height between the air distributor and tube, which should be carefully chosen to guarantee the end-point quality of product. Moreover, inappropriate setting of these parameters might lead to particle agglomeration and result in defluidization in the process. Therefore, it is necessary to monitor the complex gas-solids flows inside the bed. However, as a complicated structure, even without coating solution involved, the complex gas-solids flow behaviour is still difficult to monitor and predict [4]. The process is often operated based on "trial-and-error" method and the process is a black box [5]. Therefore, it is necessary to provide efficient measurement tools to investigate the complex flow behaviour inside the Wurster type fluidized bed and improve the process efficiency and product quality.

Currently, two methods are commonly used to monitor the coating process, i.e. single point based measurement and tomography based measurement. In the single point measurement, pressure transducer or optical probes are used to investigate the solids concentration and fluctuations. The pressure signals carry the information such as turbulence intensity, particle movement and eruption of bubbles and such information can be retrieved by signal processing both in time domain and frequency domain analysis [6]. However, pressure signals can only estimate the global information, for example solids concentration. Optical probe is another option to measure particles concentration and velocity based on the signal reflection and attenuation $[7,8]$. Other single point based probes, including capacitance and piezoelectric probe, were reported for the measurement of gas-solids flow $[9,10]$.

Compared with the single point based measurement, process tomography is one of the best options to monitor the gassolids flow process in a Wurster type fluidized bed due to the nature of its non-intrusive and non-invasive. Until now, several type of process tomography have been applied in the investigation of gas-solids fluidized bed, including electrical capacitance tomography (ECT) [11-13], microwave tomography (MWT) [14] and magnetic resonance imaging (MRI) [15]. Among the above process tomography technologies, ECT is the best choice for the application in fluidized bed coating process due to the high speed data acquisition and it can reconstruct the permittivity distribution. Normally, ECT is used in a conventional chamber with either square or circular shapes. The complicated structure of Wurster tube in a fluidized bed makes it is difficult to apply conventional sensors to measure the process. Recently, an ECT sensor with 12-4-8 external-internal electrodes was reported to investigate the gas-solids fluidized bed process with Wurster tube to monitor the solids concentration $[4,16]$. However, the measurement is conducted on a single flow region either inside or outside the Wurster tube. As particles circulated between the annulus and coating zone periodically, the measurement lost the 
important information on the circulation and interaction between those regions. In terms of process control, it is also necessary to monitor the gas-solids flows in different zones synchronously and provide valuable information.

Therefore, the main objective of this research is to develop a new ECT measurement strategy to enable the synchronous measurement of the gas-solids flows both inside and outside of the Wurster tube. To achieve such objects, an ECT sensor with combined 12-4-8 electrodes was designed and reconstructed. Several sets of experiment are conducted by varying the gap height, fluidization air velocity and initial particle mass loading. In addition, a dual planes ECT sensor with eight electrodes in each plane was applied to further investigate the flow behaviours inside the Wurster tube. The flow regimes were revealed in 2D ECT images. The standard deviation and power spectra density were applied to analyse the particle fluctuation and circulation characteristics. In addition, the solids concentration and average velocity inside the Wurster tube were investigated by the dual planes ECT sensor.

\section{Experimental method}

\subsection{Test facility}

The lab-scale Wurster type fluidized bed is depicted in Fig. 1. The main frame of the fluidized bed consists of a plenum in the bottom, a conical chamber with a height of $350 \mathrm{~mm}$, an expansion chamber with a height of $500 \mathrm{~mm}$ and a filter on the top. The conical chamber and the expansion chamber are made of Plexiglas, which enable the visual observation of the particle flow. The thickness of chamber wall is $0.5 \mathrm{~cm}$. The ECT sensor and Wurster tube are installed in the conical chamber. The diameter and the length of Wurster tube is $65 \mathrm{~mm}$ and $150 \mathrm{~mm}$ respectively. An air distributor made of stainless steel located 5 20 mm under the Wurster tube, a locking device is used to adjust the gap between the air distributor and Wurster tube. A pair of pressure transducers (Keller PD23) are used to acquire the pressure drop in the annulus zone. In order to control the flow pattern under the Wurster tube, the open areas of holes in the air distributor are $35 \%$ in central region and $4 \%$ in annular region respectively. A roots blower is used to adjust the fluidization air velocity.

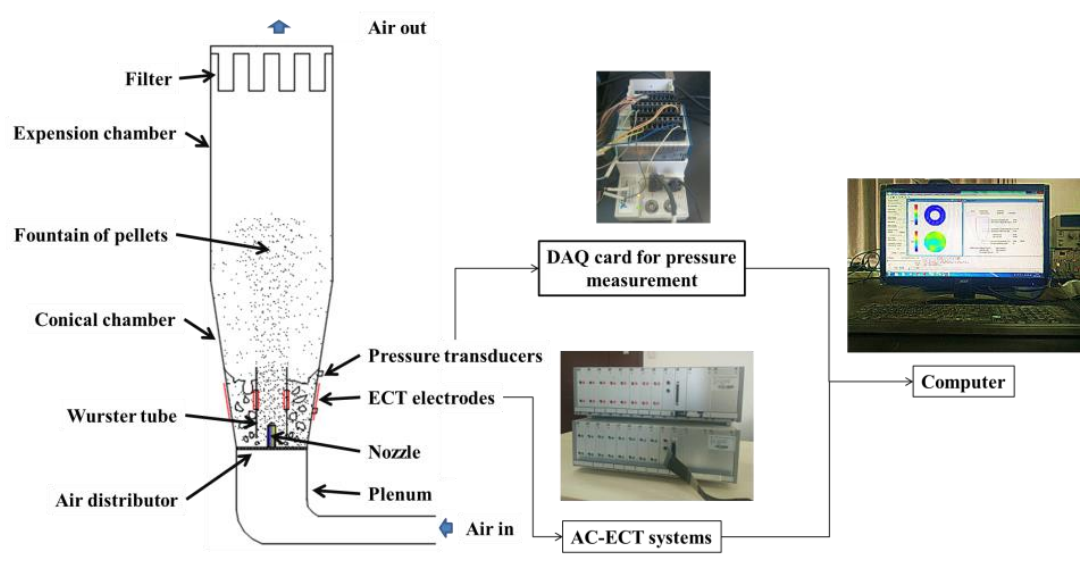

Fig. 1 Schematic of Wurster type fluidized bed and measurement instrumentations 


\subsection{ECT sensor and image reconstruction}

The structure of combined 12-4-8 electrodes and dual planes ECT sensor are given in Fig. 2. The 12-4 combined electrodes (E1 E16) are used to measure the annulus zone and the rest 8 electrodes (E17 E24) to measure the coating zone inside Wurster tube. The Wurster tube is made of stainless steel to act as a shielding layer. The cross section being measured is in the range of $6 \mathrm{~cm}$ to $11 \mathrm{~cm}$ above the bottom of the Wurster tube, and each pixel of the ECT image depicts an axial average value in that region. The excitation frequency is in the range of $50 \mathrm{kHz}$ to $250 \mathrm{kHz}$ and the voltage amplitude up to $20 \mathrm{~V}$. The total number of measured capacitance is 120 for the $12-4$ combined electrodes and 28 for the 8 electrodes for ECT sensor respectively.
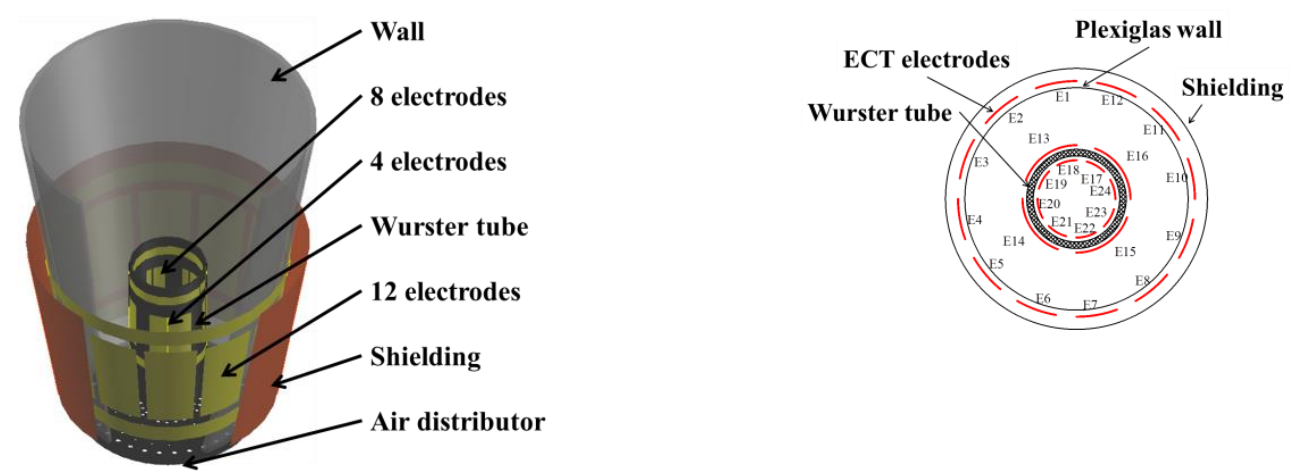

(a) Combined 12-4-8 electrodes ECT sensor
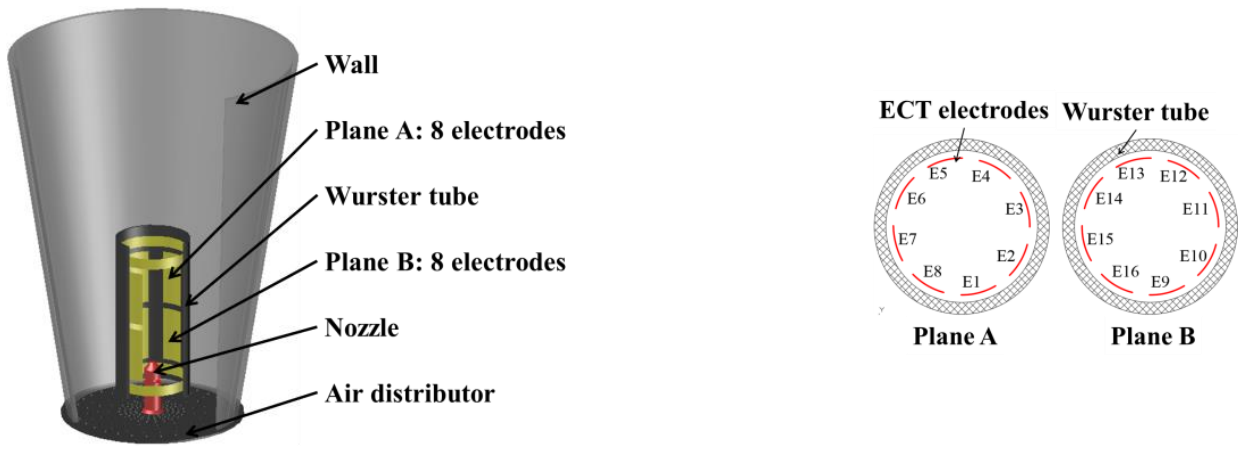

(b) Dual planes ECT sensor

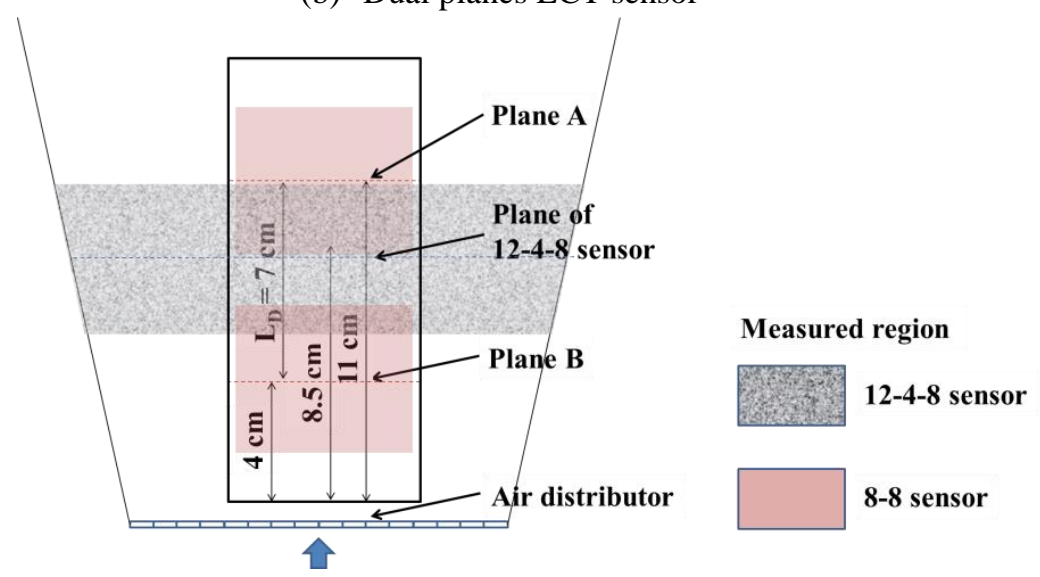

(c) The measured regions of ECT sensors

Fig. 2 Structure of ECT sensors 
For the dual planes ECT sensor, the measurements in the two planes are synchronously. The dual planes being measured located $1.5 \sim 6.5 \mathrm{~cm}$ and $8.5 \sim 13.5 \mathrm{~cm}$ above the bottom of the Wurster tube, respectively. The total number of measured capacitances is 56 .

The image reconstruction is based on the sensitivity maps $\boldsymbol{S}$ and the measured capacitances. The sensitivity maps were obtained by COMSOL simulation. The element size and exciting sequences in the simulation were the same with the measurement. In the simulation, the relative permittivity inside chamber was fixed to 1.0 and the permittivity of the chamber wall was set to 3.0. The sensitivity maps are calculated by

$$
S_{i j}(P)=-\iint_{\sigma_{p}} \frac{\nabla \varphi_{\mathrm{i}}(x, y)}{V_{\mathrm{i}}} \cdot \frac{\nabla \varphi_{j}(x, y)}{V_{\mathrm{j}}} d x d y
$$

where $S_{i j}(P)$ is the sensitivity value between the $\mathrm{i}^{\text {th }}$ and $\mathrm{j}^{\text {th }}$ electrodes over one pixel area $\sigma_{p}, \varphi_{i}(x, y)$ is the potential distribution when the electrode $i$ is excited with a voltage of $V_{\mathrm{i}}$.

The Landweber iteration [17] is used to reconstruct the ECT image and the algorithm is written as

$$
\boldsymbol{G}^{n+1}=\boldsymbol{G}^{n} \cdot(1+\lambda)+\alpha \cdot \boldsymbol{S}^{T} \cdot\left(\boldsymbol{C}_{N}-\boldsymbol{S} \cdot \boldsymbol{G}^{n}\right) \cdot(1-\lambda)
$$

where $\alpha$ is the step length, $\lambda$ is the relaxation factor and $\mathrm{n}$ is the iteration number, $\boldsymbol{G}$ is the normalized grey level, which donates the normalized permittivity distribution in the cross section area. The value of step length are chosen based on the a optimum method [18]. However, there are no general rules for choosing the best value for the relaxation factor. It is believed that the optimum value depends on a number of factors, e.g. the nature of the problem, the number of grid points, the grid spacing, and the iteration procedure used [19]. In this research, the value of $\lambda$ is set to zero. $\boldsymbol{C}_{\mathrm{N}}$ in equation (2) is the normalized capacitance vector. The normalized capacitance is obtained by

$$
C_{\mathrm{N}}(k)=\frac{\left(\boldsymbol{C}_{\mathrm{M}}-\boldsymbol{C}_{\mathrm{L}}\right)_{k}}{\left(\boldsymbol{C}_{\mathrm{H}}-\boldsymbol{C}_{\mathrm{L}}\right)_{k}}
$$

where $\boldsymbol{C}_{\mathrm{H}}$ and $\boldsymbol{C}_{\mathrm{L}}$ are the low and high calibration capacitance respectively, $k$ is the element number of capacitance vectors.

The average solids concentration in a specific area of cross section is linearly related to the grey level of ECT image by

$$
\beta=\vartheta \cdot \frac{\sum_{i=1}^{N} G_{i}(x, y) a_{i}(x, y)}{\sum_{i=1}^{N} a_{i}(x, y)}
$$


where the coefficient $\vartheta$ is the packing density of the solids, and it is defined as the pure solids volume in the unit bulk volume of particle assemblage. The value of $\vartheta$ is estimated to be 0.62 when the diameter ratio of the vessel to the particle is higher than 10 [20]. $a$ is the area of pixel, $N$ is the total pixels in the cross-sectional area.

\subsection{Experiment material and setting}

Sucrose pellets are used in this research and the pellets properties are given in Table 1. The manipulated parameters of the experiment include the fluidization air velocity $\left(u_{f}\right)$, the gap height between the Wurster tube and air distributor $\left(h_{g a p}\right)$ and the loading mass weight of pellets $\left(m_{b e d}\right)$. Table 2 gives all the process parameters for nine different cases. The fluidization air velocity was adjusted by changing the frequency of the blower, the fluidization air velocity ranged from $0.98 \sim 3.50$ $\mathrm{m} / \mathrm{s}$ were applied for each case. For case 2, 5 and 8, experiments were conducted by ECT sensor with two structures. In this research, all tests were "cold" flow without spraying coating solvent into the bed.

Table 1. Properties of the pellet

\begin{tabular}{cc}
\hline Material & Sucrose pellet \\
\hline Mean pellet diameter $(\mu \mathrm{m})$ & 710 \\
Apparent density $\left(\mathrm{kg} / \mathrm{m}^{3}\right)$ & 1550 \\
Mass loading $(\mathrm{kg})$ & $2 \sim 4$ \\
\hline
\end{tabular}

Table 2. Process parameters setting

\begin{tabular}{ccccc}
\hline Case No. & $m_{\text {bed }}(\mathrm{kg})$ & $h_{\text {gap }}(\mathrm{mm})$ & $u_{f}(\mathrm{~m} / \mathrm{s})$ & \multicolumn{2}{c}{ ECT sensor } \\
\hline 1 & 2 & 5 & $12-4-8$ & $8-8$ \\
\hline 2 & 3 & 5 & $\sqrt{ }$ & $\sqrt{ }$ \\
3 & 4 & 5 & $\sqrt{ }$ & \\
4 & 2 & 10 & $\sqrt{ }$ & \\
5 & 3 & 10 & $\sqrt{ }$ & $\sqrt{ }$ \\
6 & 4 & 10 & $\sqrt{ }$ & $\sqrt{ }$ \\
7 & 2 & 20 & & $\sqrt{ }$ \\
9 & 3 & 20 & & $\sqrt{ }$ \\
\hline
\end{tabular}




\section{Results and discussion}

\subsection{Static tests for $\mathbf{1 2 - 4 - 8}$ ECT sensor}

To validate the proposed ECT sensor and evaluate the image reconstruction quality, four static tests were carried out with the same test materials as the dynamic measurements. The theoretical distributions and reconstructed images are given in Fig. 3.

Theoretical distribution

Image reconstruction
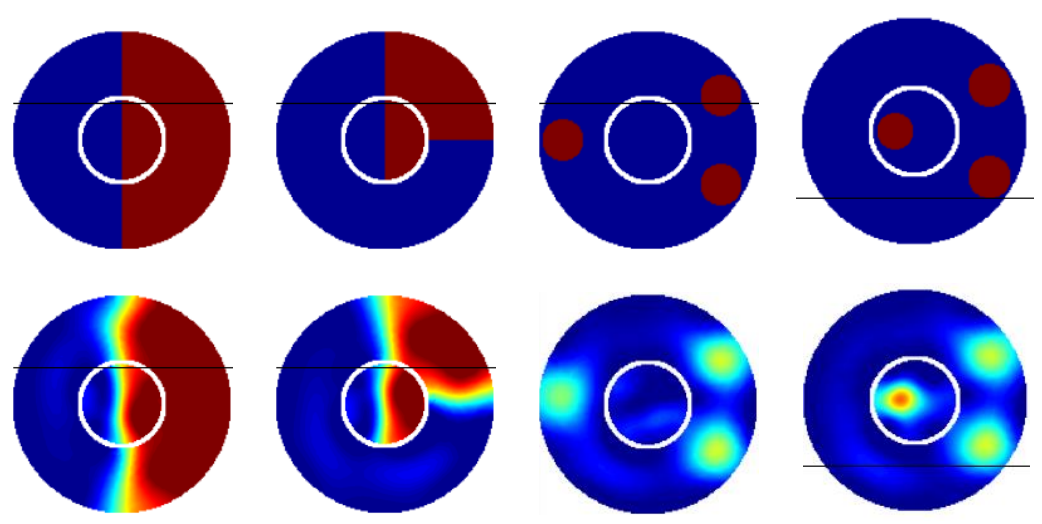



Fig. 3 Theoretical distributions and reconstructed images

To assess the correspondence between the theoretical distribution and the reconstructed images, two parameters are used:

(1) correlation coefficient and (2) image error. The definitions of the two parameters are as follows.

Correlation coefficient:

$$
C_{\text {coef }}=\frac{\sum_{\mathrm{i}=1}^{\mathrm{N}}\left(\hat{G}_{\mathrm{i}}-\overline{\hat{\boldsymbol{G}}}\right)\left(G_{\mathrm{i}}-\overline{\boldsymbol{G}}\right)}{\sqrt{\sum_{\mathrm{i}=1}^{\mathrm{N}}\left(\hat{G}_{\mathrm{i}}-\overline{\hat{\boldsymbol{G}}}\right)^{2} \sum_{\mathrm{i}=1}^{\mathrm{N}}\left(G_{\mathrm{i}}-\overline{\boldsymbol{G}}\right)^{2}}}
$$

Image error:

$$
\gamma=\frac{\|\hat{\boldsymbol{G}}-\boldsymbol{G}\|}{\|\boldsymbol{G}\|} \times 100 \%
$$

where $\hat{\boldsymbol{G}}$ and $\boldsymbol{G}$ are the assumed and reconstructed permittivity distributions, respectively.

Table 3 summarizes the correlation coefficient and image error for four static tests. It can be seen that the proposed ECT sensors can reconstruct the assumed permittivity distribution with relatively low image errors. More information about the validation of ECT model for gas-solids flow measurement can be found in references [21]. The dual planes ECT sensor consists of two planes and the sensor structure of each plane is same as the 8 electrodes in above 12-4-8 electrodes ECT sensor. The validation of the eight electrodes sensor can be found in reference [22]. 
Table 3. Correlation coefficient and image error for static tests

\begin{tabular}{ccccc}
\hline Distribution & 1 & 2 & 3 & 4 \\
\hline$C_{\text {coef }}$ & 0.9528 & 0.9397 & 0.7401 & 0.7763 \\
$\gamma$ & 0.2195 & 0.2628 & 0.6475 & 0.6185 \\
\hline
\end{tabular}

\subsection{Flow regimes identification in a Wurster type fluidized bed}

Fig. 4 shows the image reconstruction results with different experiment settings and fluidization air velocity. Fig. 5 gives the mean solids concentration profiles in the radical direction calculated from the time-series ECT images in Fig. 4. The ECT images clearly show the dilute phase flow in the coating zone and dense phase flow in the annulus zone. The flow is nearly kept in a minimum fluidization regime in the annulus zone with low fluidization air velocity as shown in Fig. 4(a) (b). However, it is transferred into bubble flow regime with the increase in fluidization air velocity as shown in Fig. 4 (d) in the annual zone. The gas-solids flow in the coating zone is relative complex. For $h_{g a p}=5 \mathrm{~mm}$ and $10 \mathrm{~mm}$ respectively, there are 2 types of flow regimes, i.e. annular flow (Fig. 4 (a), Fig. 4 (e)) and core flow (Fig. 4 (c), Fig. 4 (d), Fig. 4 (f) Fig. 4 (h)). The flow regime transition occurs with the increase in the fluidization air velocity. For $h_{\text {gap }}=20 \mathrm{~mm}$, the annular flow can be observed (Fig. 4 (i)). With the further increase in fluidization air velocity, gas-solids flow becomes dilute and dispersed in the coating zone. The solids concentration profiles as shown in Fig. 5 clearly demonstrates the flow regime transition in the reconstructed images as shown in Fig. 4 for different fluidization velocity, gap heights and initial mass loading.

se 2

$\left(h_{\text {gap }}=5 \mathrm{~mm}\right.$, mbed $\left._{\text {be }}=3 \mathrm{~kg}\right)$

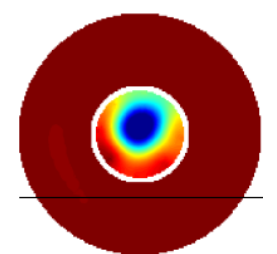

(a)

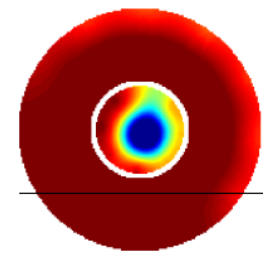

(e)

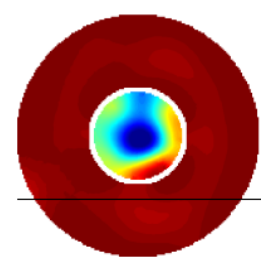

(i)

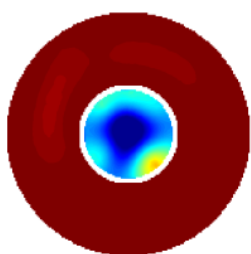

(b)

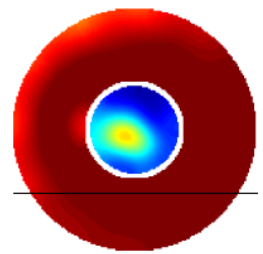

(f)



(j)



(c)

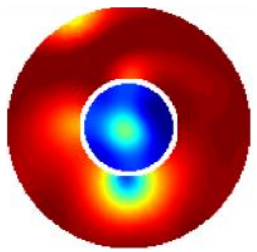

(g)

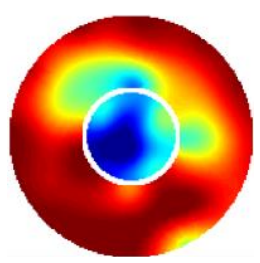

(k)

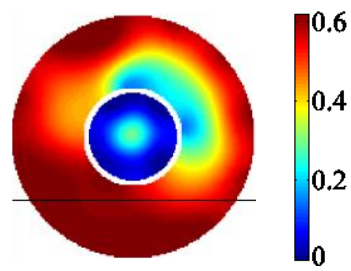

(d)

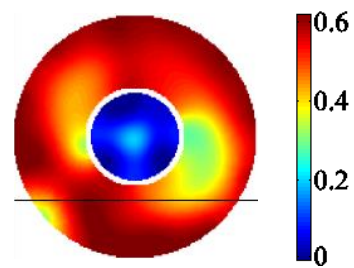

(h)

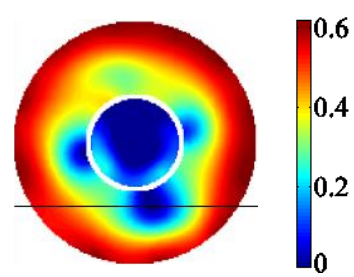

(1) $u_{f}=0.89 \mathrm{~m} / \mathrm{s}$

$u_{f}=1.18 \mathrm{~m} / \mathrm{s}$

$u_{f}=2.34 \mathrm{~m} / \mathrm{s}$

$u_{f}=3.50 \mathrm{~m} / \mathrm{s}$

Fig. 4 Reconstructed images of 12-4-8 ECT sensor 


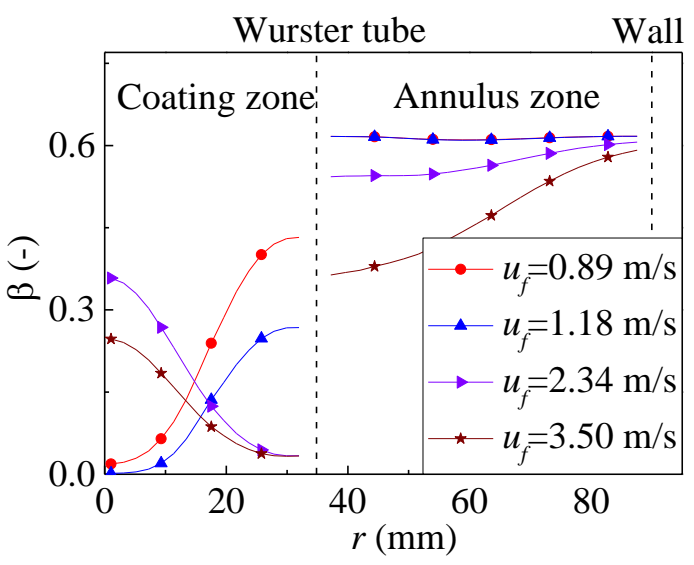

(a) Case $2\left(h_{\text {gap }}=5 \mathrm{~mm}, m_{\text {bed }}=3 \mathrm{~kg}\right)$

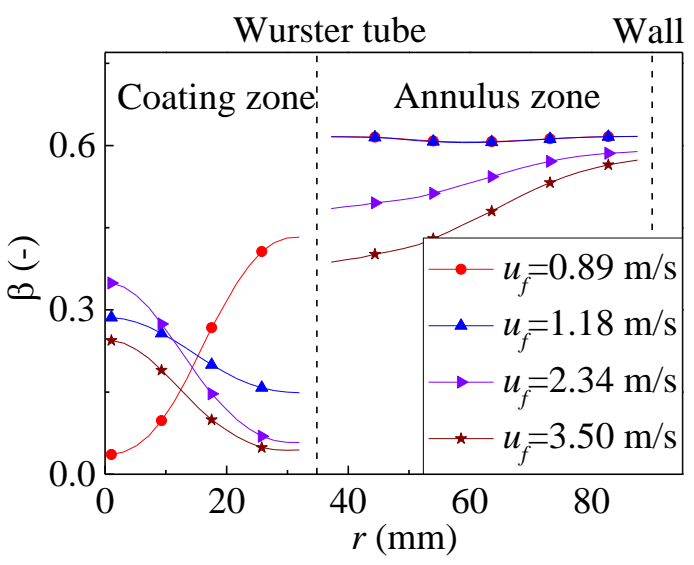

(b) Case $5\left(h_{\text {gap }}=10 \mathrm{~mm}, m_{\text {bed }}=3 \mathrm{~kg}\right)$

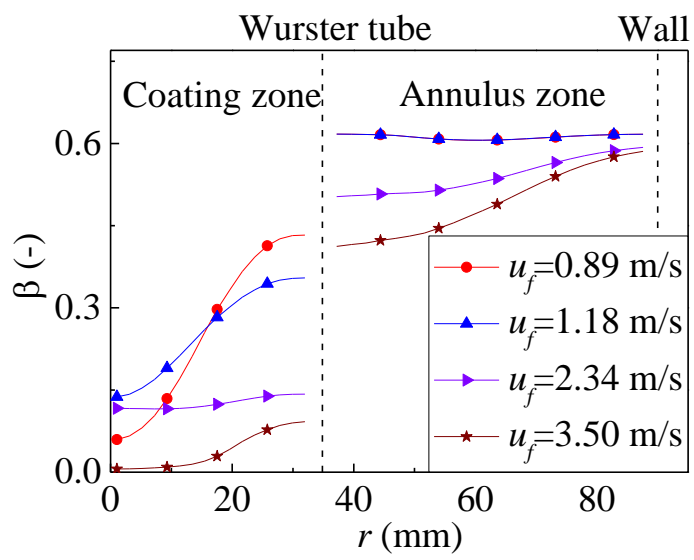

(c) Case $8\left(h_{\text {gap }}=20 \mathrm{~mm}, m_{\text {bed }}=3 \mathrm{~kg}\right)$

Fig. 5 Radial concentration profiles in the cross-sectional area

To investigate the minimum fluidized velocity $\left(u_{m f}\right)$ in the annulus zone, additional tests were carried out for each case by increasing the fluidization air velocity from 0 to $2.2 \mathrm{~m} / \mathrm{s}$ steady. Normally, $u_{m f}$ is determined by the plot of pressure drop of the bed as a function of fluidization air velocity [23]. Unlike a conventional bubbling bed, the chamber of Wurster type fluidized bed consists of two zones, and its fluidization process is more complex compared with the conventional one. Fig. 6 shows the pressure and averaged solids concentration change with time for case 1 (similar results can also be found in other cases). The solids concentration is the average value of all pixels of reconstructed image in the coating or annulus zone. The thin lines are smooth lines with low-pass filter. As can be seen from Fig. 6, there are three stages during the increase in the fluidization air velocity. In the first stage, the air velocity is very low while the pressure rises rapidly and it is still a fixed-bed condition. In the second stage, the pressure has a sharp decrease. In this stage, the pellets in the coating zone are fluidized which can be seen from the reconstructed ECT images. After that point, the pressure increases slowly with the increase in the fluidization air velocity and the solids concentration decreases rapidly in the coating zone while keeps constant in the annulus zone. It also can be seen from ECT images that the flow regime in the coating zone transfers 
from annular to core flow. In the third stage, the bed is in fluidization and the pressure nearly keeps constant. In this research, the minimum fluidization velocity is determined when solids concentration in the annulus zone starts to flow. However, the pressure in the bed does not change obviously when this fluidization happens. One possible reason is that the pressure drop across the annulus zone was balanced by the pressure inside the Wurster tube. Fig. 7 shows the $u_{m f}$ of different cases based on the above analysis. In general, the value of $u_{m f}$ decreases with the increase in gap height, and the value of $u_{m f}$ for initial mass loading of $2 \mathrm{~kg}$ is lower than that for the cases of $3 \mathrm{~kg}$ and $4 \mathrm{~kg}$.

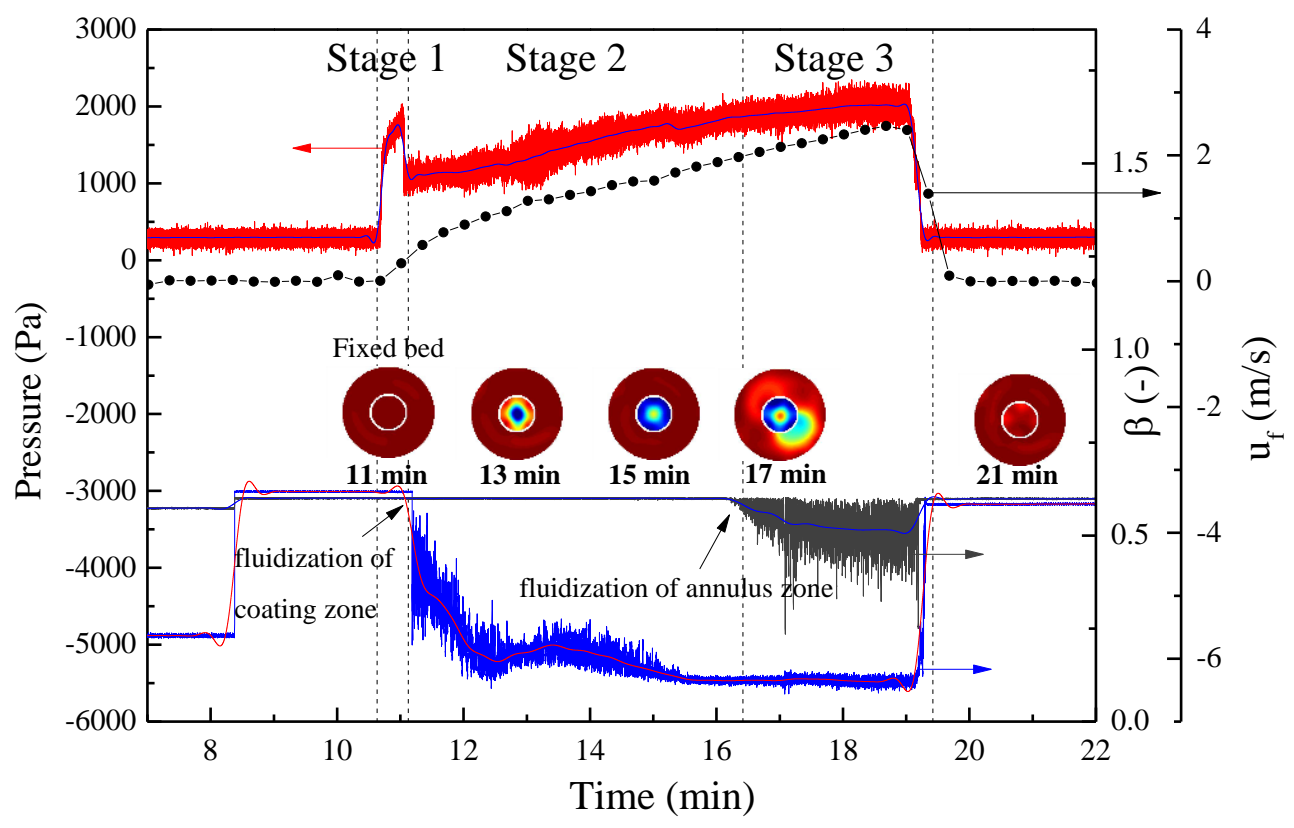

Fig. 6 Variation in pressure and solids concentration

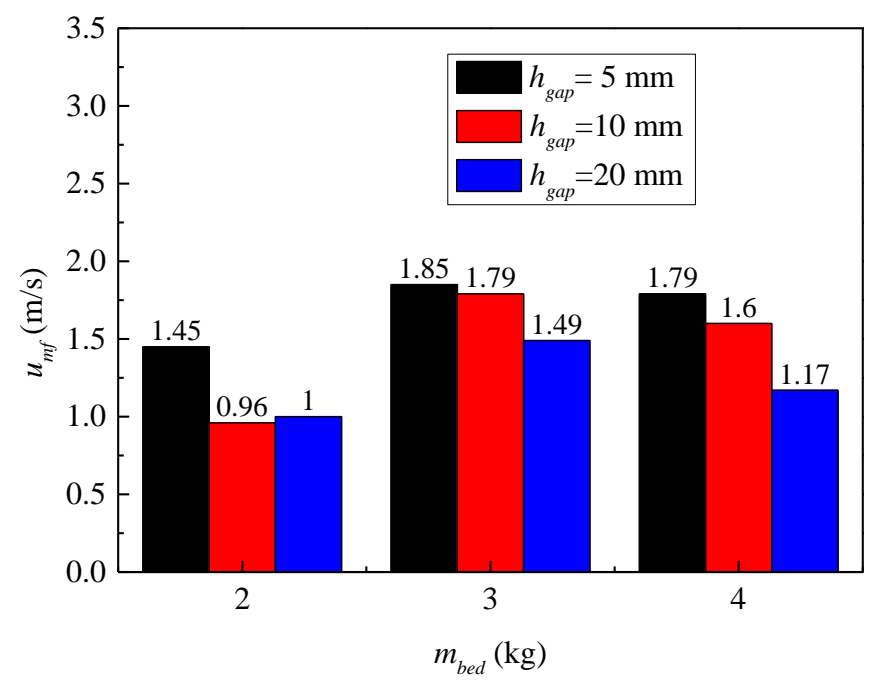

Fig. 7 Minimum fluidized velocity for different cases

Fig. 8 compares the solids concentration with different mass loadings and gap heights. In this figure, the concentration value was obtained by time-averaged over 2000 frames with the same operation condition. Generally for all cases, the solids concentration decreases with the increase in fluidization air velocity. For $h_{g a p}=10 \mathrm{~mm}$ and $5 \mathrm{~mm}$, a sharp decrease 
in the coating zone can be found with the fluidization velocity of $1.5 \mathrm{~m} / \mathrm{s}$. After that point, the decrease of solids concentration becomes relative slow. For $h_{g a p}=20 \mathrm{~mm}$, the solids concentration in the coating zone decreases with the fluidization air velocity linearly.

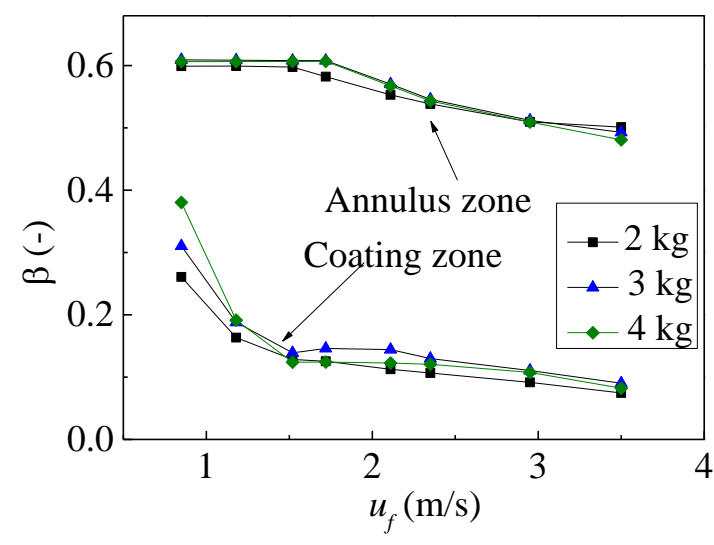

(a) $h_{g a p}=10 \mathrm{~mm}$

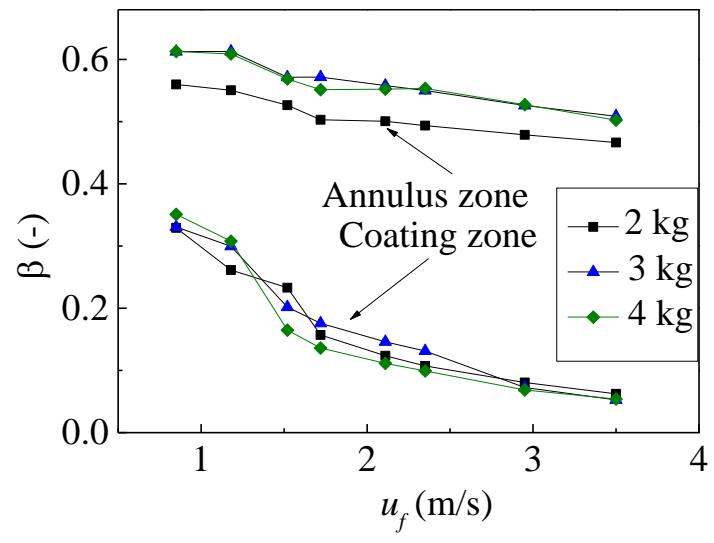

(b) $h_{\text {gap }}=20 \mathrm{~mm}$

Fig. 8 Variation in solids concentration with the fluidization air velocity and mass loadings

Fig. 9 shows the solids concentration variation under different gap heights. In the annulus zone, the turning point of concentration changes with different gap setting which indicates that pellets start to be fluidized. The fluidization air velocity corresponding to the turning point decreases with the increase of gap height, which is consistent with the result in Fig. 7.

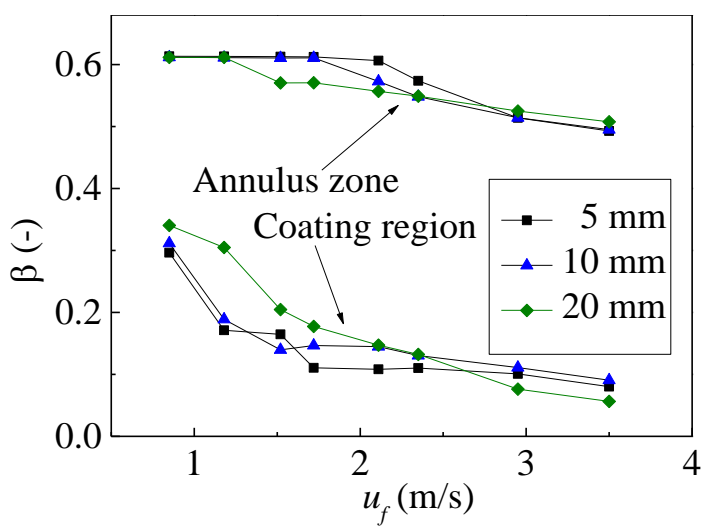

Fig. 9 Variation in solids concentration with the fluidization air velocity and gap heights

\subsection{Fluctuation of solids concentration}

Power spectra density (PSD) is a normal method to analysis the time-series signals fluctuation of gas-solids flow in frequency domain, it is obtained by Fast Fourier Transform (FFT) analysis [24]. Fig. 10 gives the variation of time-series solids concentration and the corresponding PSD spectrum with different operation conditions. With low fluidization velocity of $1.71 \mathrm{~m} / \mathrm{s}$ (case 2), the intensity of fluctuation is very small and the PSD distribution is very smooth in both zones. With the fluidization air velocity increasing to $2.34 \mathrm{~m} / \mathrm{s}$, bubbles are generated in the annulus zone (Fig. 7), and the gas-solids flows start to fluctuate. When fluidization air velocity increases to $3.50 \mathrm{~m} / \mathrm{s}$, there are one dominant frequency 
of $2.2 \mathrm{~Hz}$ in the coating and annulus zones. For the case 9 with the fluidization air velocity of $2.13 \mathrm{~m} / \mathrm{s}$, there are two dominate frequencies $(0.81 \mathrm{~Hz}$ and $1.73 \mathrm{~Hz})$ in the annulus zone while only one $(1.73 \mathrm{~Hz})$ is in the coating zone. This means that the link between the annulus and coating zone is very weak and the flow is separated in the bed with low fluidization velocity. When fluidization air velocity increases to $3.50 \mathrm{~m} / \mathrm{s}$, there are more dominate spectrum in both zones and they have nearly the same frequency components, which indicates that the flow is linked together in the annulus and coating zone and the fluctuation is synchronously with high fluidization velocity.
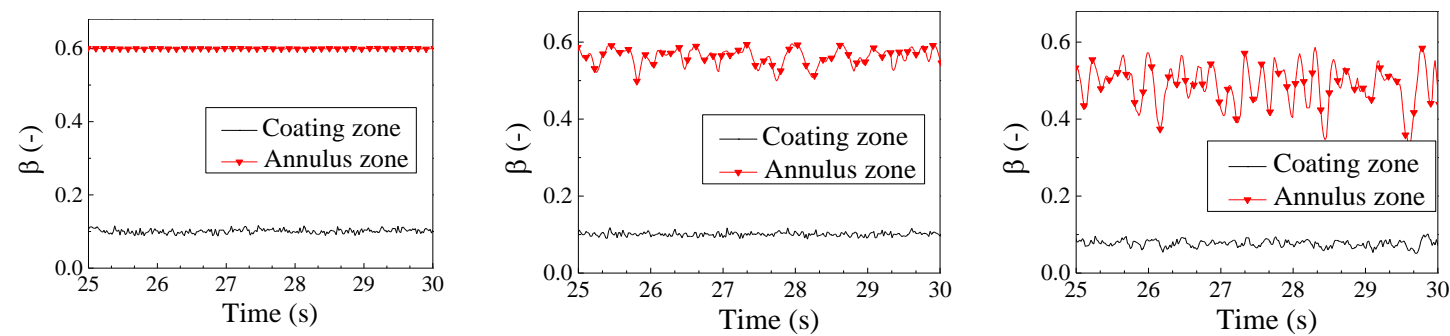

Case 2
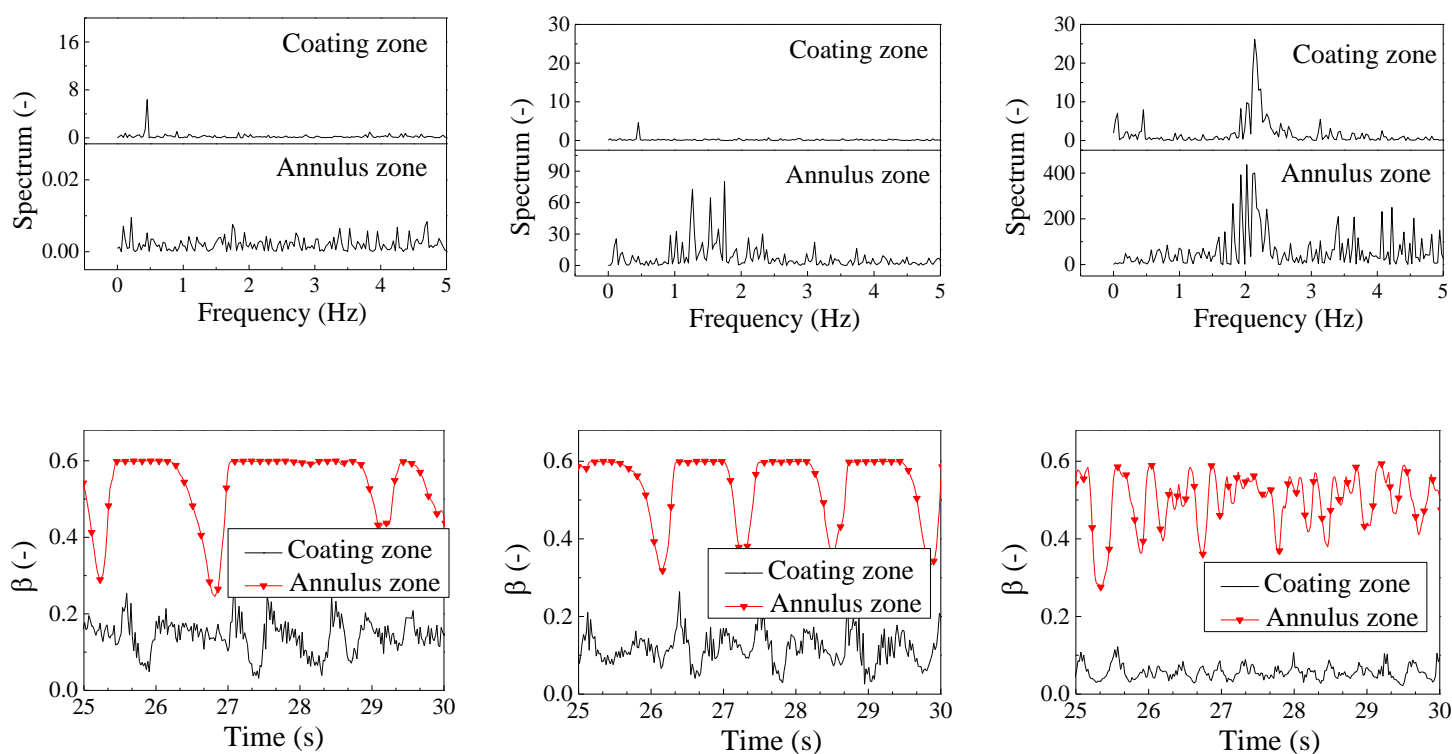

Case 9


$u_{f}=1.71 \mathrm{~m} / \mathrm{s}$

$u_{f}=2.34 \mathrm{~m} / \mathrm{s}$

$u_{f}=3.50 \mathrm{~m} / \mathrm{s}$

Fig. 10 Variation of solids concentration both in time and frequency domain 
Fig. 11 gives the standard deviation $(S D)$ of solids concentration both in the coating and annulus zone for different cases.. As can be seen, the value of $S D$ for the annulus zone increases with the increase of fluidization air velocity, the main reason is that with the fluidization of annulus zone pellets, bubbles are generated and enhance the local concentration fluctuation. For the cases of $h_{g a p}=5 \mathrm{~mm}$ and $10 \mathrm{~mm}$, the value of $S D$ in the coating zone decrease sharply with the velocity range of $0 \sim 1.5 \mathrm{~m} / \mathrm{s}$, and after that, $S D$ keeps stable in a low level. However, for the case of $h_{g a p}=20 \mathrm{~mm}$, the $S D$ values in the coating zone are obviously high and it has a similar tendency with that in the annulus zone. It should be noted that the flow regime in the coating zone transferred to core flow (see Fig. 4) for the cases of $h_{g a p}=5 \mathrm{~mm}$ and $10 \mathrm{~mm}$ and transferred to dispersed flow for the case of $h_{\text {gap }}=20 \mathrm{~mm}$. It indicates that core flow is one type of steady flow which can stabilize the pellet fluctuation. In terms of process optimization, the cases of $h_{g a p}=5 \mathrm{~mm}$ and $10 \mathrm{~mm}$ are better because the gas-solids flow keeps steady in the coating zone even with a high fluidization air velocity. Further, the fluidization air velocity should not be very high, to avoid bubble generation in the annulus zone and interrupt the downwards particle flow [25].

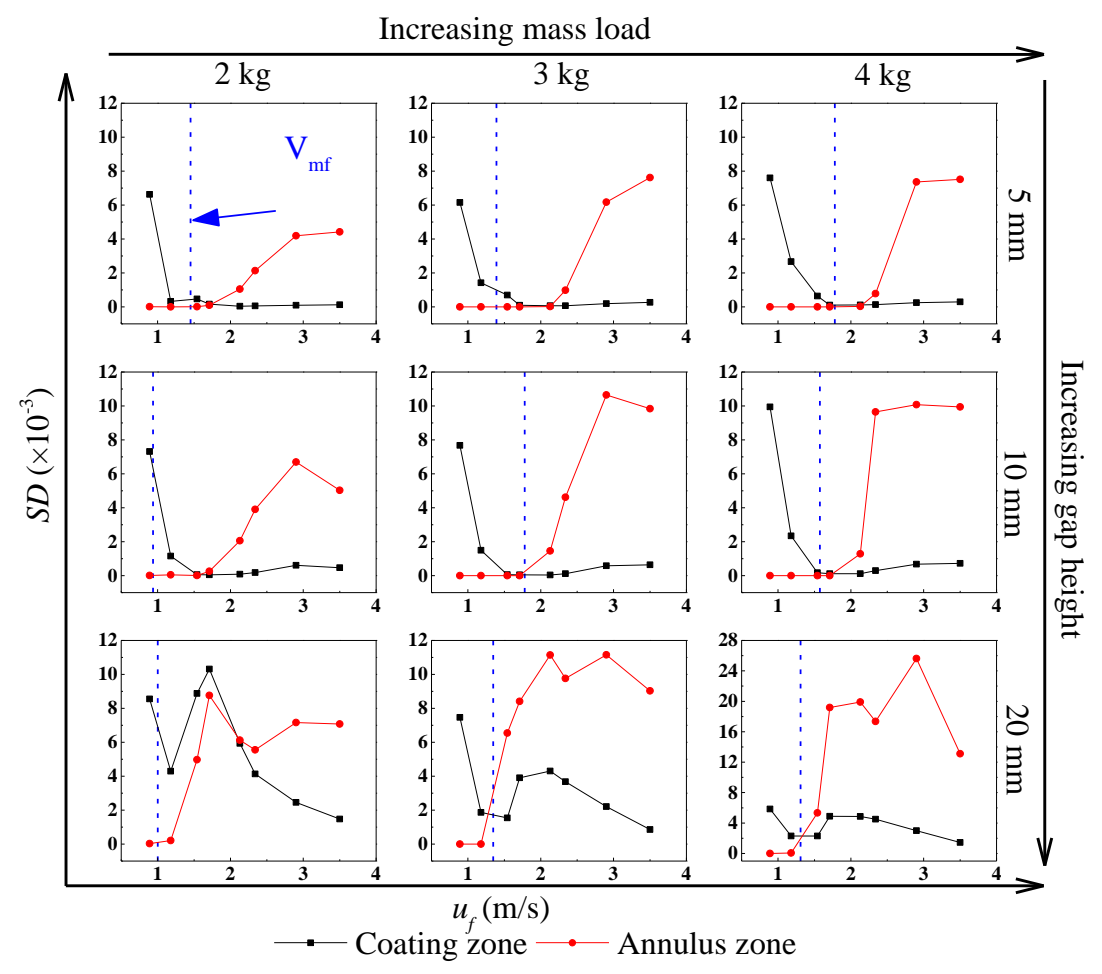

Fig. 11 Variation in $S D$ of pellet concentration with different operation conditions

\subsection{Solids concentration inside the Wurster tube by dual planes ECT sensor}

It is important to study the gas-solids flow inside the Wurster tube, as pellets are wetted by solution droplets in this region. Fig. 12 gives the reconstructed images by the dual planes ECT sensor as shown in Fig. 2 (c). As can be seen from those images, for the fluidization air velocity of $0.98 \mathrm{~m} / \mathrm{s}$, the gas-solids flow is still a dense phase flow inside the Wurster tube. With the increase of fluidization air velocity to $1.54 \mathrm{~m} / \mathrm{s}$, core flow can be seen for both planes with $h_{g a p}=10 \mathrm{~mm}$. For the case of $h_{\text {gap }}=20 \mathrm{~mm}$, the flow in plane A turns to dispersed flow while the flow in plane B turns to unsteady core flow. By associating ECT images in Fig. 12 with ECT images obtained by 12-4-8 ECT sensor in Fig. 4, it can be concluded that the 
gas-solids flow regime is always core flow inside the Wurster tube for the case of $h_{\text {gap }}=10 \mathrm{~mm}$. However, for the case of $h_{\text {gap }}=20 \mathrm{~mm}$, the core flow is only found in the bottom of the Wurster tube.

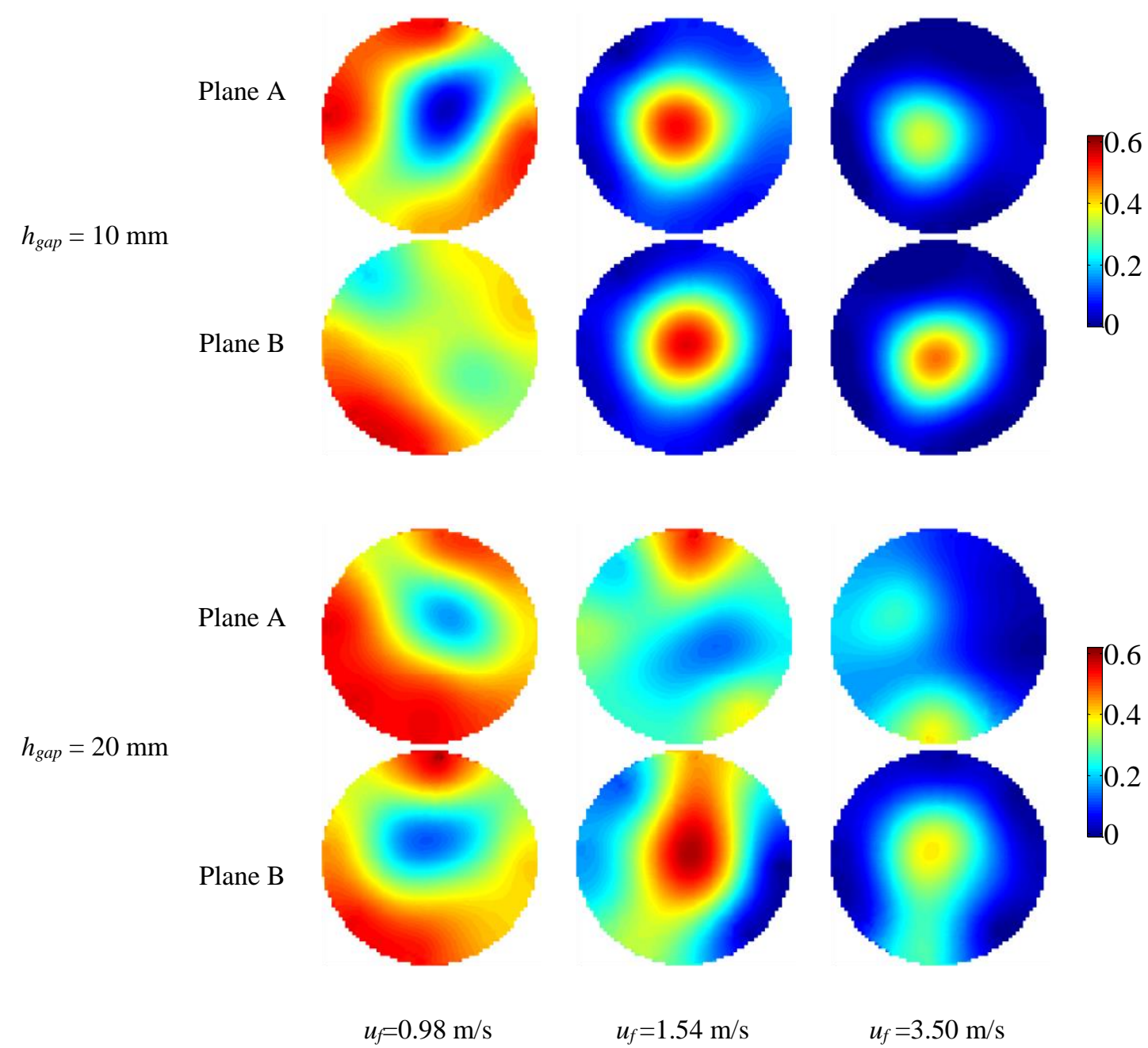

Fig. 12 Dual plane ECT images

Fig. 13 gives several samples of solids concentration variation in the dual planes. Fig. 14 shows the PSD spectrum corresponding to Fig. 13. It can be seen from Fig. 13 and Fig. 14 that the mean solids concentration and the concentration fluctuation frequency of plane A and plane B are very similar with each other, which imply that the gas-solids flow is relevant in those two planes. 

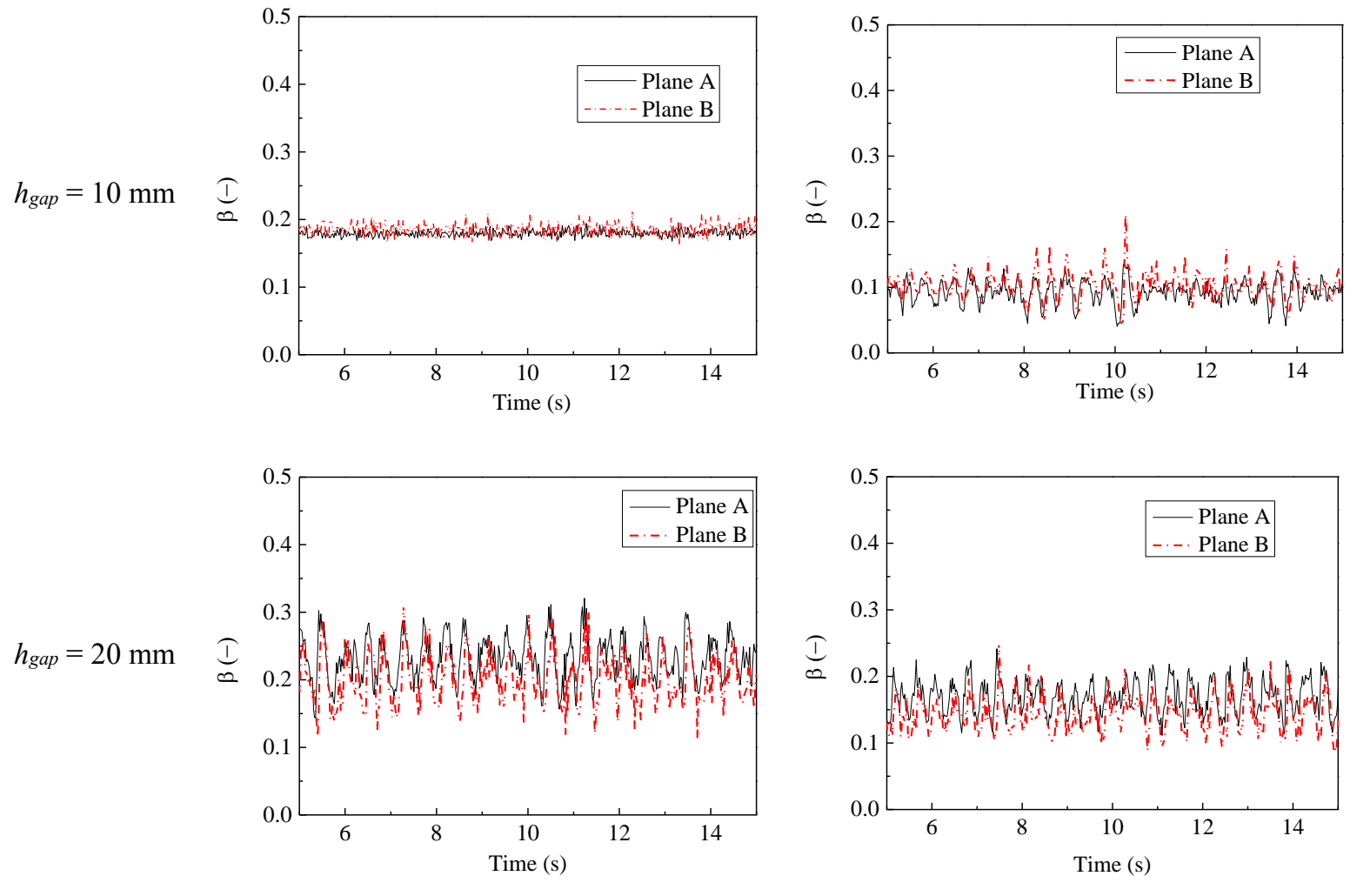

$u_{f}=2.13 \mathrm{~m} / \mathrm{s}$

$u_{f}=2.90 \mathrm{~m} / \mathrm{s}$

Fig. 13 Variation of solids concentration in time domain
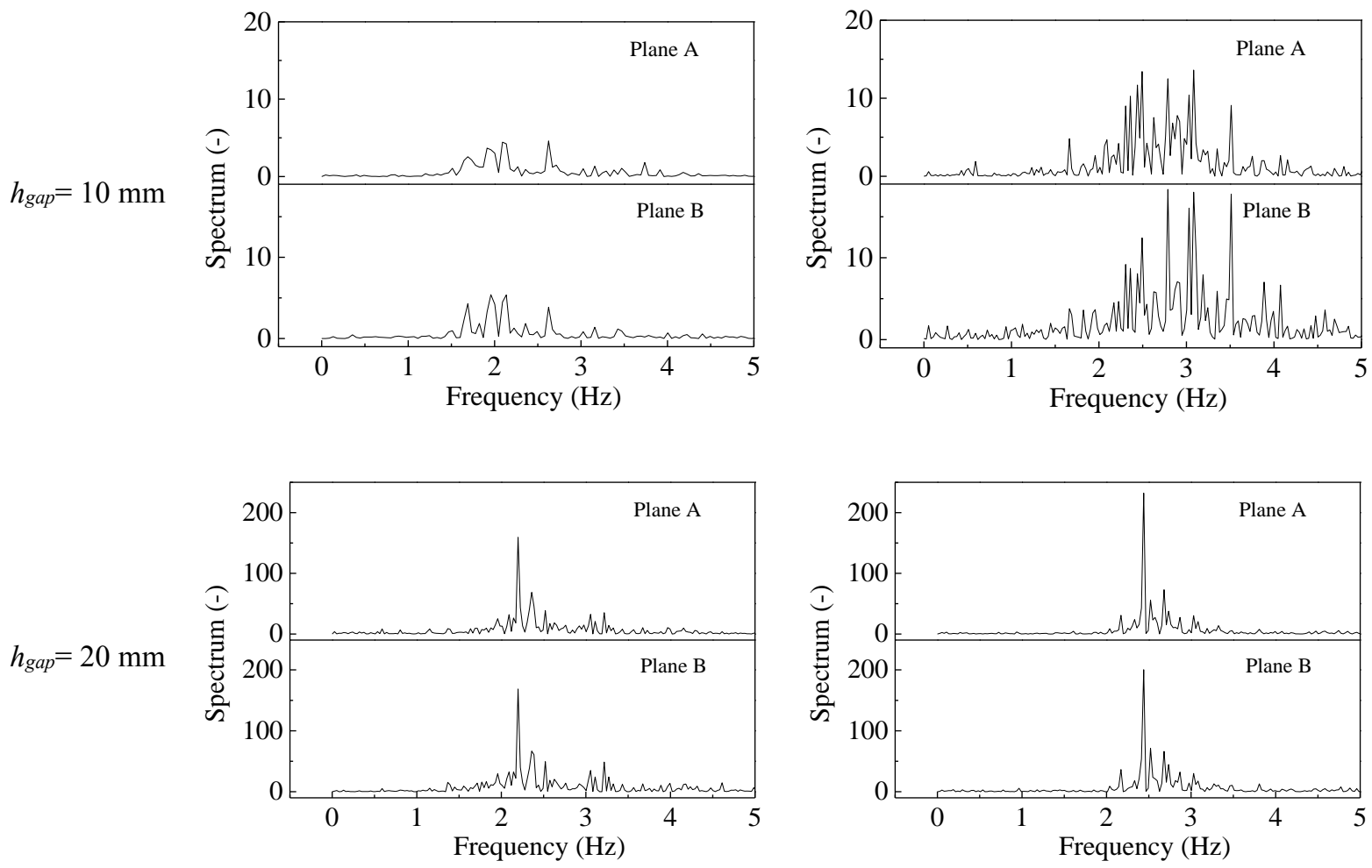

$u_{f}=2.13 \mathrm{~m} / \mathrm{s}$

$$
u_{f}=2.90 \mathrm{~m} / \mathrm{s}
$$


Fig. 14 PSD spectrum of solids concentration

Fig. 15 shows the variation in $S D$ of solids concentration for the above cases, the $S D$ values measured by 12-4-8 ECT sensor ( Fig. 11) under the same operation parameter are also illustrated for comparison. The case of $h_{\text {gap }}=10 \mathrm{~mm}$ gives lower $S D$ value than that of $h_{g a p}=20 \mathrm{~mm}$. It can also be seen from this figure that the $S D$ value of plane A is lower than that of plane B for both cases, which indicates that the gas-solids flow turns unsteady with the increase in the height of plane inside the Wurster tube. Compared the $S D$ curves by the 12-4-8 and dual planes ECT sensor with the same operation condition, both measurements give similar trends.

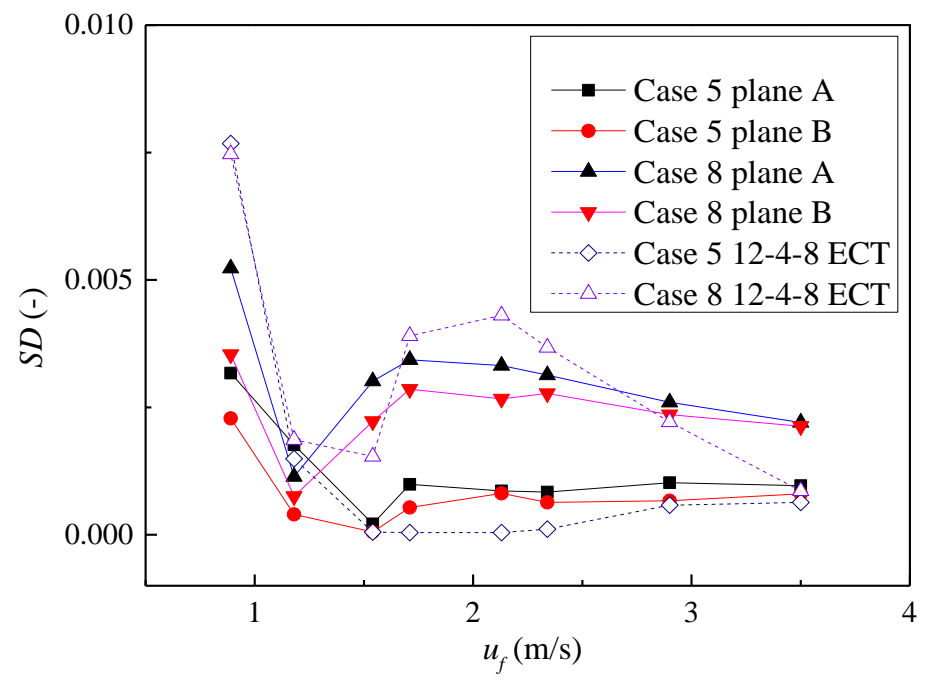

Fig. 15 Variation in $S D$ of solids concentration inside Wurster tube

\subsection{Solids velocity measurement inside the Wurster tube}

The solids velocity in fluidized bed is normally measured by the particle imaging [26] or particle tracking [27] methods. In this research, the solids velocity inside Wurster tube is measured by the cross-correlation method [28]. This method based on the measurement of two sensors placed along the solids flow direction with a short distance, and the sensors sample synchronously. When solids flow by, the "tagging" signals generated by flow turbulence or suspended moving solids are recorded by the sensors one after another. The signal structures recorded by different sensors are similar due to the short distance between them, and this method also requires a high data acquisition speed for both sensors. The delay time between the two signals is estimated using cross-correlation coefficient, and then the flow velocity between the two sensors can be obtained based on the delay time and the distance between sensors. The measured velocity is the average velocity within both temporal and spatial regions. By this method, the capacitance signals from multi-plane structured ECT sensor have been successfully applied to measure the bubble rising velocity or solids velocity in a fluidized bed [29-31]. The length of electrodes is relative large, however, when solids are passing though a plan (from the bottom to the top of an electrode), the capacitance value also changes (the solids flow is not uniform), hence the unique "tagging" signals can also be recorded by the sensor. 
For the measured time-series capacitance signals $C_{A}$ and $C_{B}$ from plane $\mathrm{A}$ and $\mathrm{B}$, the cross-correlation coefficient is calculated by [32]

$$
R_{A B}(j)=\frac{1}{N} \sum\left(C_{B}(i)-\bar{C}_{B}\right) \cdot\left(C_{A}(i+j)-\bar{C}_{A}\right), j=0,1,2, \cdots, N-1
$$

where $N$ is the sample number, and it is $900, j$ is the delay frame number. In the Eq. (7), the capacitances used for calculation include adjacent electrode pairs (E1-E2 from plane A and E9-E10 from plane B) and opposite electrode pairs (E1-E5 from plane A and E9-E13 from plane B). If $j_{m}$ is the delay frames corresponds to the maximum value of $R_{A B}$, then the delay time between the two capacitance signals can be calculated by

$$
\tau_{m}=j_{m} / f
$$

where $f$ is the data acquisition speed of ECT sensor, and it is $185 \mathrm{~Hz}$ for the dual plane structure. The solids velocity inside Wurster tube is then calculated by

$$
u_{s}=L_{D} / \tau_{m}
$$

where $L_{D}$ is the distance between plane $\mathrm{A}$ and $\mathrm{B}$, and it is $7 \mathrm{~cm}$ in this research.

Fig. 16 gives the cross-correlation of adjacent electrode capacitances with different fluidization air velocity. The crosscorrelation value convergences with the increase in delay frames. The number of delay frames ranges from 5 to 9 with different air velocity, which indicates $185 \mathrm{~Hz}$ is sufficient high for solids velocity measurements. Fig. 17 gives the solids velocity change with different fluidization velocity and gap heights. The velocity calculated by cross-correlation ranges from $1.4 \mathrm{~m} / \mathrm{s}$ to $2.6 \mathrm{~m} / \mathrm{s}$ and slightly increases with the increase in fluidization air velocity. The calculated results indicate that the particles velocity is more sensitive to the fluidization air velocity and less to the gap height. The solids velocity inside the Wurster tube is rarely investigated in previous literatures, and it is determined by various operational parameters. However, the results from both CFD simulation [33] and experimental measurement [26] show the scale of solids velocity possibly lies in $1 \sim 3.5 \mathrm{~m} / \mathrm{s}$, which agree well with present measurements. In addition, the temporal resolution of ECT sensor might be improved with high data acquisition speed ECT system [34]. 

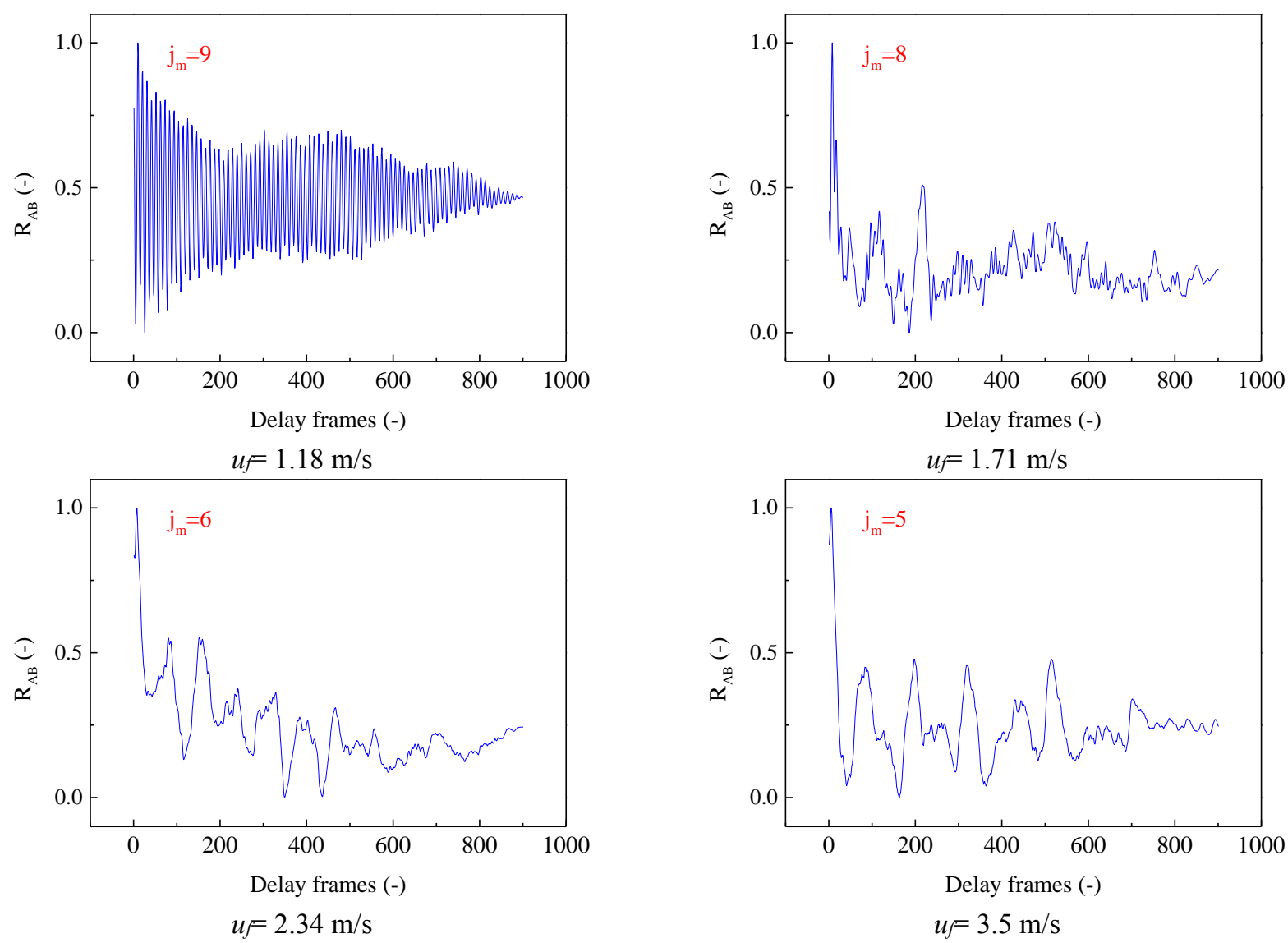

Fig. 16 Cross-correlation of adjacent electrode capacitance with different fluidization air velocity

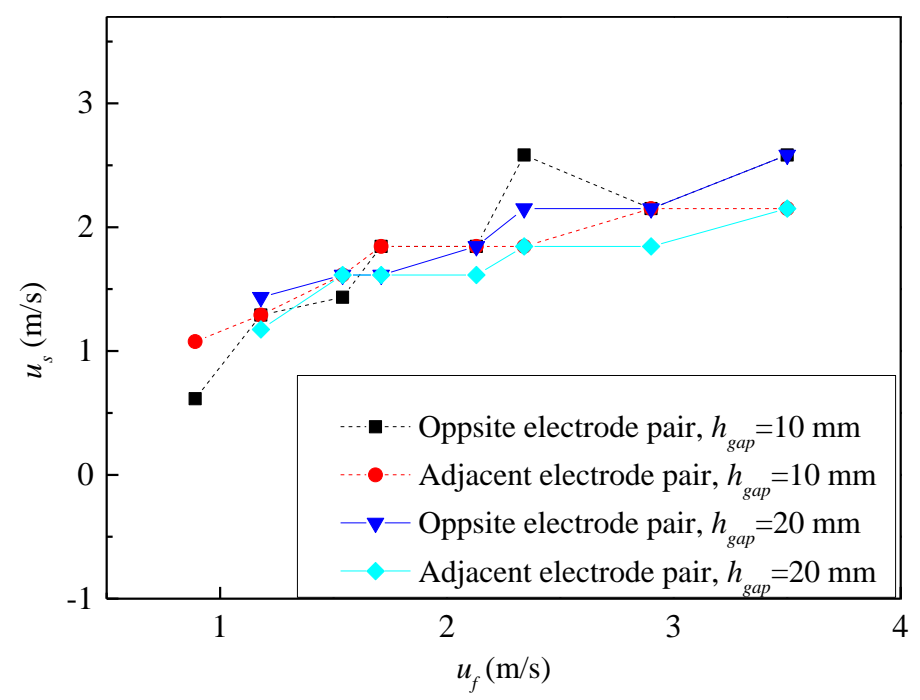

Fig. 17 Solids velocity with different fluidization air velocity 


\section{Conclusion}

This paper presented the measurement results for the gas-solids flows inside a Wurster type fluidized bed based on two types of ECT sensor. The flows both in the coating and annulus zone are measured synchronously for the first time. The main conclusions are summarized as follows.

The gas-solids flow regimes in the coating and annulus zones are revealed based on ECT images. The measurement result of 12-4-8 ECT sensor shows that the gap height between the Wurster tube and air distributor has an obvious effect on the flow regimes in the bed. Generally, the annulus zone is occupied by minimum fluidization flow regime and bubble flow regime. The coating zone is occupied by annular, core and dispersed flows, which depend on the gap height, fluidization velocity and initial mass loading. The measurement result from the dual planes ECT sensor further reveals that the flow regimes inside the Wurster tube are different between the two planes. With a low gap height, the flow keeps in core regime in both planes. With a high gap height, the flow turns from core flow regime in the bottom to dispersed flow regime in the upper plane.

Power spectral density analysis of solids concentration shows that the coating and annulus zone share same fluctuation frequency components especially after the pellets are fluidized in the annulus zone. Based on the standard deviation of solids concentration, it indicates that low gap height cases give the good flow stability in the coating zone, which might facilitate the coating uniformity.

The above result indicates that ECT senor serves as a powerful tool to monitor the gas-solids flow in a Wurster type fluidized bed. However, the measurement conducted in this research is only focused the "cold" flow without spraying coating solution and future works will focus on the investigation of experiments with solution droplets effect on the process.

\section{Acknowledgements}

The authors are grateful to the support from the National Natural Science Foundation of China (Nos. 61320106004 and 61374018) and CAS Interdisciplinary Innovation Team.

\section{References}

[1] S. Srivastava, G. Mishra, Fluid bed technology: overview and parameters for process selection, International Journal of Pharmaceutical Sciences and Drug Research 2010; 2:236-246.

[2] D.E. Wurster, Air-suspension technique of coating drug particles - a perliminary report, Journal of the American Pharmaceutical Association 1959;48:451-454.

[3] K. KuShaari, P. Pandey, Y. Song, R. Turton, Monte Carlo simulations to determine coating uniformity in a Wurster fluidized bed coating process, Powder Technology 2006;166:81-90. 
[4] H. Wang, G. Qiu, J. Ye, W. Yang, Experimental study and modelling on gas-solids flow in a lab-scale fluidised bed with Wurster tube, Powder Technology 2016; 300:14-27.

[5] S. Shelukar, J. Ho, J. Zega, E. Roland, N. Yeh, D. Quiram, A. Nole, A. Katdare, S. Reynolds, Identification and characterization of factors controlling tablet coating uniformity in a Wurster coating process, Powder Technology 2000;110:29-36.

[6] C.A.M. da Silva, J.J. Butzge, M. Nitz, O.P. Taranto, Monitoring and control of coating and granulation processes in fluidized beds-A review, Advanced Powder Technology 2014;25:195-210.

[7] T. Ishikura, H. Nagashima, M. Ide, Hydrodynamics of a spouted bed with a porous draft tube containing a small amount of finer particles, Powder Technology 2003;131:56-65.

[8] R. Rundqvist, A. Magnusson, B.G.M. van Wachem, A.E. Almstedt, Dual optical fibre measurements of the particle concentration in gas/solids flows, Experiments in Fluids 2003;35:572-579.

[9] V. Wiesendorf, J. Werther, Capacitance probes for solids volume concentration and velocity measurements in industrial fluidized bed reactors, Powder Technology 2000;110:143-157.

[10] J.L. Spenik, J.C. Ludlow, Use of piezoelectric pressure transducers to determine local solids mass flux in the riser of a cold flow circulating fluidized bed, Powder Technology 2010;203:86-90.

[11] H. Wang, P. Senior, R. Mann, W. Yang, Online measurement and control of solids moisture in fluidised bed dryers, Chemical Engineering Science 2009;64:2893-2902.

[12] J. Zhang, M. Mao, J. Ye, H. Wang, W. Yang, Investigation of wetting and drying process in a gas-solids fluidized bed by electrical capacitance tomography and pressure measurement, Powder Technology 2016;301:1148-1158.

[13] V. Rimpiläinen, L.M. Heikkinen, M. Vauhkonen, Moisture distribution and hydrodynamics of wet granules during fluidized-bed drying characterized with volumetric electrical capacitance tomography, Chemical Engineering Science 2012;75:220-234.

[14] H. Wang, J. Zhang, M. Ramli, M. Mao, J. Ye, W. Yang, Z. Wu, Imaging wet granules with different flow patterns by electrical capacitance tomography and microwave tomography, Measurement Science and Technology 2016;27:114007.

[15] C. Müller, J. Davidson, J. Dennis, P. Fennell, L. Gladden, A. Hayhurst, M. Mantle, A. Rees, A. Sederman, Oscillations in gas-fluidized beds: Ultra-fast magnetic resonance imaging and pressure sensor measurements, Powder Technology 2007;177:87-98. 
[16] R. Ge, J. Ye, H. Wang, W. Yang, Measurement of particle concentration in a Wurster fluidized bed by electrical capacitance tomography sensors, AIChE Journal 2014;60:4051-4064.

[17] W. Yang, L. Peng, Image reconstruction algorithms for electrical capacitance tomography, Measurement Science and Technology 2002;14:R1-R13.

[18] S. Liu, L. Fu, W.Q. Yang, Optimization of an iterative image reconstruction algorithm for electrical capacitance tomography, Measurementence \& Technology 10(7) (1999) 1970-1980.

[19] H. Wang, W. Yang, Measurement of fluidised bed dryer by different frequency and different normalisation methods with electrical capacitance tomography, Powder Technology, 2010;199:60-69.

[20] M. Fayed, L. Otten, Handbook of powder science \& technology, Springer Science \& Business Media, 2013;97-102

[21] Y.T. Makkawi, P.C. Wright, Electrical capacitance tomography for conventional fluidized bed measurementsremarks on the measuring technique, Powder Technology, 2004;148:142-157.

[22] H. Wang, Y. Li, G. Qiu, G. Song, W. Yang, Measurement of gas-solids flow in loop seal and external heat exchanger in a circulating fluidized bed, Powder Technology, 2014; 266: 249-261.

[23] O. Jaiboon, B. Chalermsinsuwan, L. Mekasut, P. Piumsomboon, Effect of flow pattern on power spectral density of pressure fluctuation in various fluidization regimes, Powder Technology, 2013; 233:215-226.

[24] O.-a. Jaiboon, B. Chalermsinsuwan, L. Mekasut, P. Piumsomboon, Effect of flow pattern on power spectral density of pressure fluctuation in various fluidization regimes, Powder Technology, 2013; 233: 215-226.

[25] L.K. Wang, P.W.S. Heng, C.V. Liew, Classification of annular bed flow patterns and investigation on their influence on the bottom spray fluid bed coating process, Pharmaceutical Research, 2010; 27: 756-766.

[26] M. Börner, M. Peglow, E. Tsotsas, Derivation of parameters for a two compartment population balance model of Wurster fluidised bed granulation, Powder Technology, 2013;238: 122-131.

[27] S. Karlsson, I. Niklasson Björn, S. Folestad, A. Rasmuson, Measurement of the particle movement in the fountain region of a Wurster type bed, Powder Technology, 2006; 165:22-29.

[28] L. Fan, T.C. Ho, W. Walawender, Measurements of the rise velocities of bubbles, slugs and pressure waves in a gas solid fluidized bed using pressure fluctuation signals, AIChE Journal, 1983;29: 33-39.

[29] S. Liu, Q. Chen, H.G. Wang, F. Jiang, I. Ismail, W.Q. Yang, Electrical capacitance tomography for gas-solids flow measurement for circulating fluidized beds, Flow Measurement and Instrumentation, 2005; 16:135-144. 
[30] G. Qiu, J. Ye, H. Wang, W. Yang, Investigation of flow hydrodynamics and regime transition in a gas-solids fluidized bed with different riser diameters, Chemical Engineering Science, 2014;116:195-207.

[31] M. Mao, J. Ye, H. Wang, W. Yang, Investigation of gas-solids flow in a circulating fluidized bed using 3D electrical capacitance tomography, Measurement Science and Technology, 2016;27:095401.

[32] S.H. Sheen, A.C. Raptis, Active ultrasonic cross-correlation flowmeters for mixed-phase pipe flows, ISA Trans.; (United States), 1985;24:53-58.

[33] S. Heinrich, M. Dosta, S. Antonyuk, Chapter Two - Multiscale Analysis of a Coating Process in a Wurster Fluidized Bed Apparatus, in: B.M. Guy, L. Jinghai (Eds.) Advances in Chemical Engineering, Academic Press, 2015; pp. 83135 .

[34] W. Yang, Design of electrical capacitance tomography sensors, Measurement Science \& Technology, 2010;21: 447453. 